\title{
PEEP Generated by High-Flow Nasal Cannula in a Pediatric Model
}

\author{
Beverly D Ejiofor, Ryan W Carroll, William Bortcosh, and Robert M Kacmarek
}

\begin{abstract}
BACKGROUND: High-flow nasal cannulas (HFNC) have been increasingly used in the pediatric critical care patient population. There are different theories about the mechanism by which HFNC reduces work of breathing, including diminishing upper airway dead space by the washout of carbon dioxide. However, one of the likely primary mechanisms by which HFNC reduces work of breathing is by generating PEEP. There are limited data assessing the PEEP delivered by moderate flows $(8-50 \mathrm{~L} / \mathrm{min})$ of HFNC, which are used most commonly in pediatric patients. METHODS: Pediatric upper-airway models were created with 5 different nares produced by a 3-dimensional printer and connected to a lung simulator. Age-specific flows were delivered via the 5 different setups. Pressure throughout the simulated airway was measured at HFNC flows of 6-60 L/min with $25 \%, 50 \%$, and $75 \%$ air leak to simulate open-mouth breathing. RESULTS: PEEPs of $1.2-36 \mathrm{~cm} \mathrm{H}_{2} \mathrm{O}$ were generated with HFNC flows of 6-60 L/min. In general, for each specific cannula, increasing the flow and decreasing the air leak resulted in higher levels of PEEP delivered $(P<.001$ and $>10 \%$ difference). Changes in lung mechanics as generated by the lung simulator to simulate different patient ages resulted in the establishment of different levels of PEEP. CONCLUSIONS: HFNCs deliver varying amounts of PEEP at the alveolar level with flows of 6-60 L/min. Increasing flow and decreasing leak resulted in the generation of greater PEEP. PEEP levels differed across cannulas and model weights at the same leak level, likely due to differences in the nasal interface between the HFNC device and the model nares. Key words: noninvasive ventilation; ventilation; high-flow nasal cannula; PEEP; child; pediatric intensive care units. [Respir Care 2019;64(10):1240-1249. (C) 2019 Daedalus Enterprises]
\end{abstract}

\section{Introduction}

High-flow nasal cannula (HFNC) therapy has recently become an alternative form of respiratory support for critically ill patients. ${ }^{1-3}$ It is well tolerated and user-friendly,

All of the authors are affiliated with Massachusetts General Hospital, Boston, Massachusetts. Drs Carroll and Kacmarek are also affiliated with Harvard Medical School, Boston, Massachusetts.

Dr Kacmarek has disclosed a relationship with Medtronic, Orange Medical, and Venner Medical. The other authors have disclosed no conflicts of interest.

Supplementary material related to this paper is available at http:// www.rcjournal.com.

Correspondence: Beverly D Ejiofor, Massachusetts General Hospital, Respiratory Care Services, 275 Cambridge Street, Boston, MA 02114. E-mail: bejiofor@partners.org

DOI: $10.4187 /$ respcare. 06470 which has resulted in its increased use worldwide. ${ }^{2-6}$ It is thought that HFNC's major impact on the work of breathing stems from generating some level of PEEP..$^{1,5-11} \mathrm{HFNC}$ may also improve respiratory mechanics by causing unidirectional gas flow in the upper airway, decreasing dead space, and washing out carbon dioxide, subsequently reducing the need for elevated minute ventilation, which ultimately reduces the work of breathing while enhancing oxygen delivery. ${ }^{1,2,5,6}$ As a result of the increased air flow entering the upper airway and enhanced carbon dioxide washout, the $\mathrm{F}_{\mathrm{IO}_{2}}$ delivered to the lower respiratory tract more closely approximates the set $\mathrm{F}_{\mathrm{IO}_{2}}$ delivered by the HFNC device. ${ }^{1-3,5,6}$ Data exist for both neonates and adults describing the PEEP generated by HFNC using relatively lower flows at $2-8 \mathrm{~L} / \mathrm{min}$ and higher flows at $60 \mathrm{~L} / \mathrm{min}$, respectively. ${ }^{1,5-11}$ However, there is a paucity of data describing the pressures generated by moderate flows (ie, $8-50 \mathrm{~L} / \mathrm{min}$ ), which are used in the majority of pediatric patients 1 month to $18 \mathrm{y}$ old. A recent study by Nielsen et $\mathrm{al}^{10}$ reports the generation of PEEP by HFNC with flows 
of up to $40 \mathrm{~L} / \mathrm{min}$ using preterm, term neonate, toddler, small child, and adult airway models, with $0 \%$ and 50\% airway leaks. ${ }^{10}$ Their data demonstrated that HFNC can generate PEEP of approximately $6-20 \mathrm{~cm} \mathrm{H}_{2} \mathrm{O}$. However, they did not assess PEEP generated by the full range of flows used in pediatric patients and young adults, nor at $25 \%$ or $75 \%$ leak. ${ }^{10}$ In addition, our study investigated a different cannula (Comfort Flo, Teleflex Morrisville, North Carolina) and our institutionally built system.

Measuring intrathoracic pressure with esophageal manometry is considered the accepted standard for measuring pleural pressure in a spontaneously breathing patient. ${ }^{1,5-9,11-16}$ This approach may be feasible in neonates and adults, but it is difficult to implement in children admitted to pediatric ICUs. Children in this age group, especially the youngest, do not tolerate a nasogastric tube. ${ }^{17}$ Inserting a nasogastric tube in an agitated patient may lead to gagging, coughing, and aspiration. ${ }^{17-19}$ This ultimately increases resistance and, therefore, the work of breathing, making any study of improved respiratory mechanics challenging. ${ }^{20}$ Sedating a child to accommodate esophageal manometry is also suboptimal. Furthermore, obtaining consent from family members and acceptance by providers is understandably problematic.

The purpose of this study was to quantify the effect of the Comfort Flo HFNC system on airway pressures in various simulated spontaneously breathing lung models with different leak conditions at a range of flows typically used for pediatric patients. We hypothesized that the PEEP level would increase with increasing flow in each model and decrease as system leak increased.

\section{Methods}

\section{Experimental Setup and Apparatus}

The study used the ASL 5000 Breathing Simulator (version 3.5, IngMar Medical, Pittsburgh, Pennsylvania) as the lung model. The ASL 5000 was programmed to simulate 7 patient models with different lung mechanics based on age. The specific ages programmed were: 1 month, 6 month, $1 \mathrm{y}, 3 \mathrm{y}, 6 \mathrm{y}, 12 \mathrm{y}$, and $18 \mathrm{y}$. Previous bench studies, clinical studies, and pre-set lung models from the ASL 5000 lung simulator were used to determine the various respiratory settings for each model. ${ }^{11,12,15,21-28}$ Table 1 summarizes each model setting.

\section{Lung Model Settings}

For each lung model setting, the ASL 5000 ventilatory pattern was set to establish a percent active inspiration of $14.5 \%$, an end-inspiratory hold of $12 \%$, a return-to-baseline airway pressure of $17 \%$, and a $0 \%$ pause.

\section{QUICK LOOK}

\section{Current knowledge}

A high-flow nasal cannula (HFNC) demonstrably generates PEEP in neonatal and adult populations at lower flows (eg, 2-8 L/min) and higher flows (eg, $\geq 60 \mathrm{~L} / \mathrm{min}$ ). Few studies have demonstrated PEEP generation at the moderate flows (eg, 8-50 L/min) that are used commonly in pediatric patients.

\section{What this paper contributes to our knowledge}

In pediatric airway models, moderate flows delivered via HFNC generated PEEPs of 1.2-36 $\mathrm{cm} \mathrm{H}_{2} \mathrm{O}$. Increasing the flow and decreasing the air leak resulted in higher levels of delivered PEEP. Changes in lung mechanics in different pediatric airway models did not have a linear relationship with delivered PEEP.

\section{HFNC Specifications and Upper Airway Model}

Five sizes of Comfort Flo HFNCs were evaluated: infant, pediatric, adult small, adult medium and adult large. The upper airway model was developed based on the work of Sivieri et al. ${ }^{11}$ The external diameter of each HFNC's prongs at their base attachment to the delivery tubing was measured with a Mitutoyo digital caliper (Mitutoyo, Aurora, Illinois) with $0.1 \mathrm{~mm}$ resolution. Measurements were made with high flow through the cannulas to ensure accurate estimation of the cannulas' diameter during use. This measurement was made with the manufacturer's recommended flow moving though the cannulas.

The HFNC sizes and the respective flows recommended by the manufacturer are listed as follows: infant, $8 \mathrm{~L} / \mathrm{min}$; pediatric, $20 \mathrm{~L} / \mathrm{min}$; small adult, $35 \mathrm{~L} / \mathrm{min}$; medium adult, $40 \mathrm{~L} / \mathrm{min}$; large adult, $50 \mathrm{~L} / \mathrm{min}$. Based on clinical practice and the literature, ${ }^{29}$ we established flows both above and below the manufacturer's recommended flow for each cannula evaluated. A simulated upper airway was developed from Tygon tubing, and a simulated nasal passageway was created with a 3-dimensional printer (Fig. 1). The diameter of the nasal passageway was $1 \mathrm{~mm}$ wider than the size of the HFNC prongs at their base attachment to the delivery tubing. The nasal passageway was $5.0 \mathrm{~cm}$ in length and was connected to the port of a $15-\mathrm{mm}$ endotracheal tube fitting, to which simulated tracheas of various diameters and lengths were attached, depending on the model size (Table 1). A T-shaped connector and valve were inserted between the $15-\mathrm{mm}$ endotracheal tube fitting and the trachea to create leak percentages of $25 \%, 50 \%$, and $75 \%$. The trachea was then attached to the ASL 5000. 
Table 1. Lung Model Settings

\begin{tabular}{|c|c|c|c|c|c|c|c|c|}
\hline Model & $\begin{array}{l}\text { Compliance, } \\
\mathrm{mL} / \mathrm{cm} \mathrm{H}_{2} \mathrm{O}\end{array}$ & $\begin{array}{l}\mathrm{P} 100, \\
\mathrm{~cm} \mathrm{H}_{2} \mathrm{O}\end{array}$ & $\begin{array}{c}\mathrm{V}_{\mathrm{T}}, \\
\mathrm{mL} / \mathrm{kg}\end{array}$ & $\begin{array}{l}\text { Resistance, } \\
\mathrm{cm} \mathrm{H}_{2} \mathrm{O} / \mathrm{L} / \mathrm{s}\end{array}$ & $\begin{array}{l}\text { Inspiratory } \\
\text { Time, s }\end{array}$ & $\begin{array}{l}\text { Breathing } \\
\text { Frequency, } \\
\text { breaths/min }\end{array}$ & $\begin{array}{c}\text { Tracheal } \\
\text { Diameter, } \\
\mathrm{cm}\end{array}$ & $\begin{array}{c}\text { Tracheal } \\
\text { Length, } \\
\text { cm }\end{array}$ \\
\hline 1 month (4 kg) & 2 & -4.0 & 24 & 50 & 0.4 & 50 & 0.6 & 3.7 \\
\hline 6 month $(8 \mathrm{~kg})$ & 5 & -4.0 & 42 & 35 & 0.5 & 40 & 0.65 & 4.5 \\
\hline 1 y (10 kg) & 10 & -4.0 & 60 & 30 & 0.5 & 35 & 0.7 & 5.4 \\
\hline 3 y $(15$ kg) & 15 & -4.0 & 90 & 20 & 0.7 & 30 & 0.8 & 6.4 \\
\hline 6 y $(20$ kg) & 20 & -4.0 & 120 & 15 & 0.7 & 30 & 0.9 & 8.2 \\
\hline 12 y $(35 \mathrm{~kg})$ & 40 & -4.0 & 210 & 10 & 0.8 & 25 & 1.6 & 10.8 \\
\hline 18 y $(75 \mathrm{~kg})$ & 60 & -4.0 & 420 & 5 & 0.8 & 25 & 1.9 & 11.8 \\
\hline
\end{tabular}

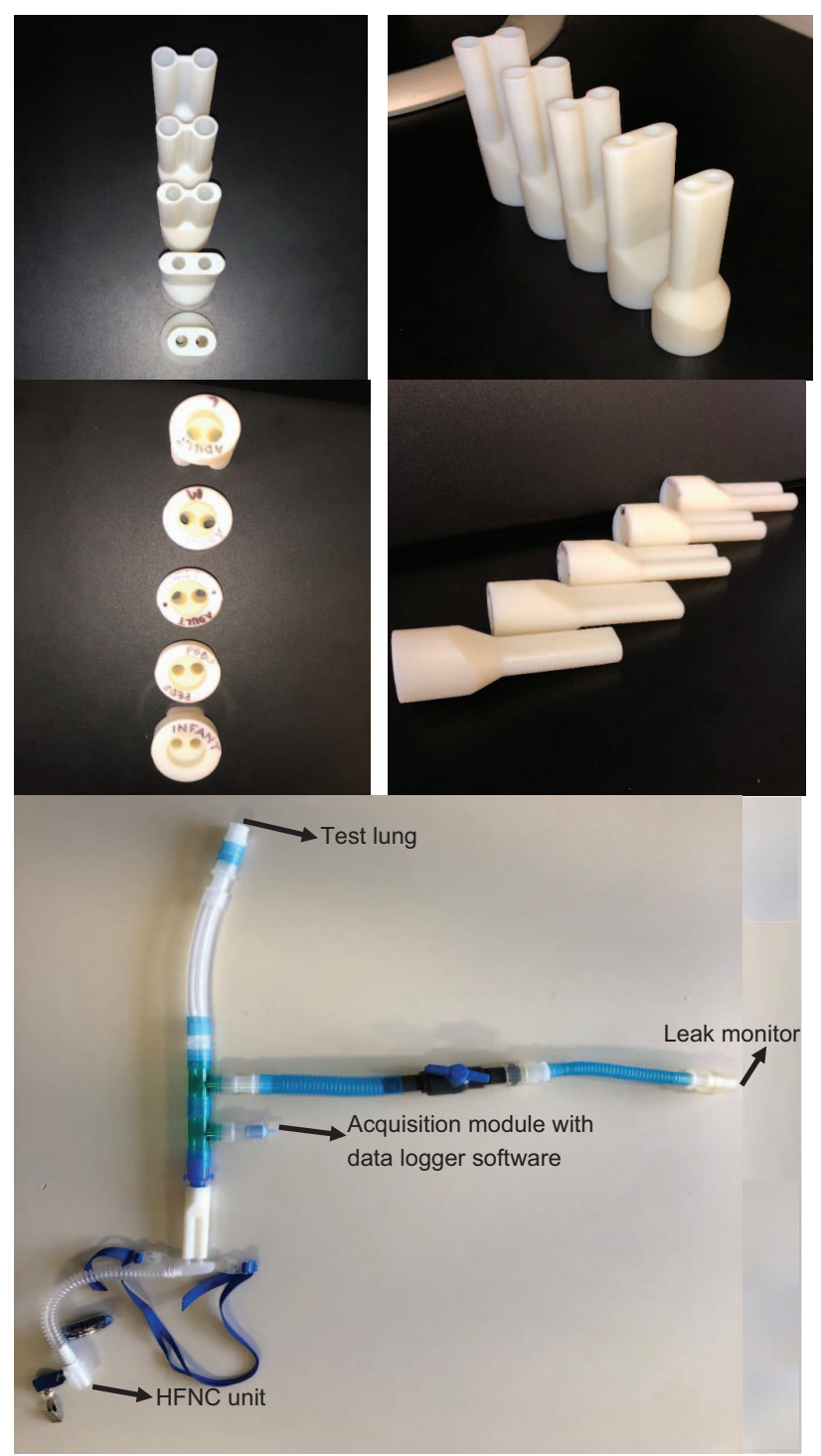

Fig. 1.5 different nares produced on a 3-dimensional printer and the high-flow nasal cannula (HFNC) experimental setup.
Table 2. Flows and Sizing for Cannula and Nares 3-Dimensional Model

\begin{tabular}{lrrlll}
\hline \hline Patient Size & \multicolumn{1}{c}{$\begin{array}{c}\text { Flow } \\
(\mathrm{L} / \mathrm{min})\end{array}$} & $\begin{array}{c}\text { Cannula } \\
\text { Sizing }\end{array}$ & $\begin{array}{c}\text { Nares } \\
\text { 3-Dimensional } \\
\text { Model }\end{array}$ \\
\hline 1 month $(4 \mathrm{~kg})$ & 6 & 8 & 10 & Infant & Infant \\
6 month $(8 \mathrm{~kg})$ & 8 & 10 & 12 & Infant & Infant \\
1 year $(10 \mathrm{~kg})$ & 15 & 20 & 25 & Pediatric & Pediatric \\
3 year $(15 \mathrm{~kg})$ & 20 & 25 & 30 & Pediatric & Pediatric \\
6 year $(20 \mathrm{~kg})$ & 25 & 35 & 45 & Adult (small) & Adult (small) \\
12 years $(35 \mathrm{~kg})$ & 30 & 40 & 50 & Adult (medium) & Adult (medium) \\
18 years $(75 \mathrm{~kg})$ & 40 & 50 & 60 & Adult (large) & Adult (large) \\
\hline
\end{tabular}

\section{HFNC Delivery System}

The HFNC delivery system used in this study was not a commercially available system, but it is the system used throughout our institution. It consisted of 2 flow meters, one for high flows and one for flows up to $15 \mathrm{~L} / \mathrm{min}$, and both were connected to a gas blender (Care Fusion, San Diego, California). Gas leaving the blender was analyzed by a oxygen analyzer (MSA, Pittsburgh, Pennsylvania). Gas then entered a Humidifier (Teleflex, Morrisville, North Carolina) and exited via the Comfort Flo HFNC system to the upper-airway lung model.

The HFNC system (both air and oxygen) was connected to an air compressor (Siemens, Lancaster, Pennsylvania), which provided the various flows associated with each cannula and patient model (Table 2). Depending on the lung model, flows of $6-60 \mathrm{~L} / \mathrm{min}$ were established. A pressure tap was inserted into the gas delivery line approximately midway between the nasal prongs and the gas source to allow for measurement of system pressure (Fig. 2 , intra-cannula pressure). A second pressure tap was placed just before the leak connection to determine the pressure drop across the simulated upper airway (Fig. 2, upper airway pressure). The humidifier was not activated to avoid 


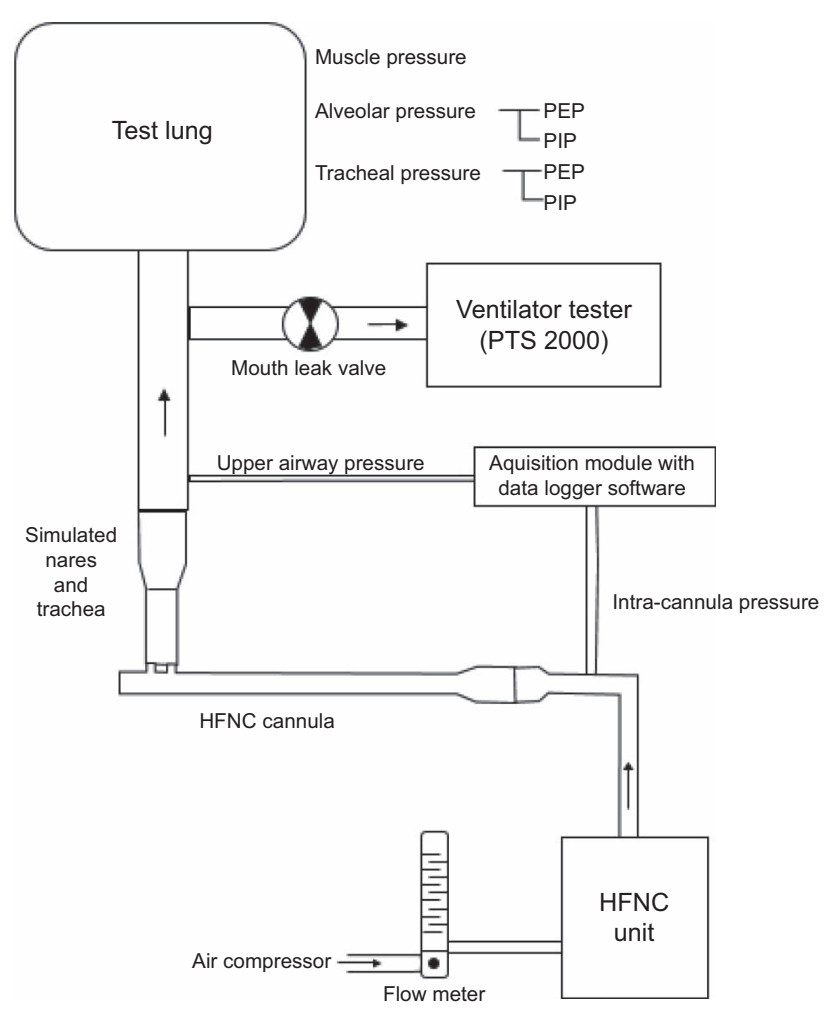

Fig. 2. Schematic of HFNC testing system. Pressure measurements indicated are shown in the online supplementary materials (Tables S1 and S2). PEP = peak expiratory pressure, PIP = peak inspiratory pressure, HFNC = high-flow nasal cannula.

accumulation of water in the computerized simulator. Thus, the system was run at room temperature.

\section{Protocol}

The specific pressures measured within the HFNC system during each trial were muscle pressure (equivalent to pleural pressure generated by the simulator), alveolar pressure (peak inspiratory, peak expiratory, and end-expiratory pressure), tracheal pressure, upper airway pressure, and intra-cannula pressure (Fig. 2). Each cannula and lung model was evaluated with different leak settings and HFNC flows as outlined in Table 2. Each configuration was tested once. After establishment of the specific flow and leak in each model, pressures were recorded for 1 minute after a 5-min stabilization period. Leak flow and percentage of total system flow were determined with a PTS 2000 ventilator tester (Puritan-Bennett Mallinckrodt, Carlsbad, California) attached to the leak port. Pressure data were collected at $200 \mathrm{~Hz}$, recorded on computers, and saved for later analysis.

The ASL 5000 measured muscle pressure, peak inspiratory pressure, peak expiratory pressure, end-expiratory pressure, alveolar pressure, and tracheal pressure (Fig. 2).
An acquisition module with DATAQ Data Logger software (DATAQ, Akron, Ohio) was used to measure pressure in the HFNC delivery tubing (intra-cannula pressure) and at the upper airway (Fig. 2). The leak created in the circuit system was measured with a PTS 2000 ventilator tester. Data from the acquisition module and the ventilator tester were time synchronized with the ASL 5000. Measurement of peak, minimum, and mean pressures were determined from all breaths during each recording period.

\section{Statistical Analysis}

Peak, minimum, and mean muscle, alveolar and tracheal pressure data were collected using the IngMar Medical ASL 5000 software. DATAQ Data Logger software was used to collect intra-cannula and upper airway pressure data. Results are expressed as mean values $\pm \mathrm{SD}$. The data were analyzed using analysis of variance to compare mean PEEP level across varying leaks within each flow and across cannulas at the same leak percentage. PEEP was determined with a $99.9 \%$ CI. Statistical analysis was conducted using Analysis Tool Pak (Excel 2016, Microsoft, Redmond, Washington) and SPSS 23.0 (SPSS, Illinois, Chicago). Significance was set at a $P<.001$, and only results that differed by $>10 \%$ were considered clinically important.

\section{Results}

There was an overall increase in end-expiratory alveolar pressure as flow increased and as leak decreased. Figure 3 shows the effect of the flow on end-expiratory alveolar pressure in each leak simulation for all models. The HFNC flow generated higher airway pressure in the 1- and 3-yold models than in the 18-y-old model. The 3-y-old model showed the greatest incremental rise in PEEP. PEEP decreased by $18.3 \mathrm{~cm} \mathrm{H}_{2} \mathrm{O}(99.9 \% \mathrm{CI} \mathrm{18-18.7)}$ as the leak increased from $25 \%$ to $50 \%$, and by $26.5 \mathrm{~cm} \mathrm{H}_{2} \mathrm{O}(99.9 \%$ CI 26.1-26.7) as the leak increased from $25 \%$ to $75 \%$. The overall difference in PEEP with the different leaks of all models is presented in Table 3 . Of note, the pressures were excessive and unreadable in the 18 -y-old model with a flow of $60 \mathrm{~L} / \mathrm{min}$ and $25 \%$ leak (Fig. 3).

Increases in leak decreased the end-expiratory alveolar pressure. Higher end-expiratory alveolar pressures were observed in the $25 \%$ and $50 \%$ leak trials than in the $75 \%$ leak trial (Fig. 3). At 75\% leak, the maximum end-expiratory alveolar pressure generated was $10.1 \mathrm{~cm} \mathrm{H}_{2} \mathrm{O}$ at $30 \mathrm{~L} / \mathrm{min}$ in the 3 -y-old model. In the other models, increasing HFNC flow with a $75 \%$ leak resulted in endexpiratory alveolar pressure of $2-7 \mathrm{~cm} \mathrm{H}_{2} \mathrm{O}$. The highest end-expiratory alveolar pressure was generated in the 3-year-old model with $25 \%$ leak, reaching $36 \mathrm{~cm} \mathrm{H}_{2} \mathrm{O}$. A rapid alveolar pressure increase was noted, especially with 

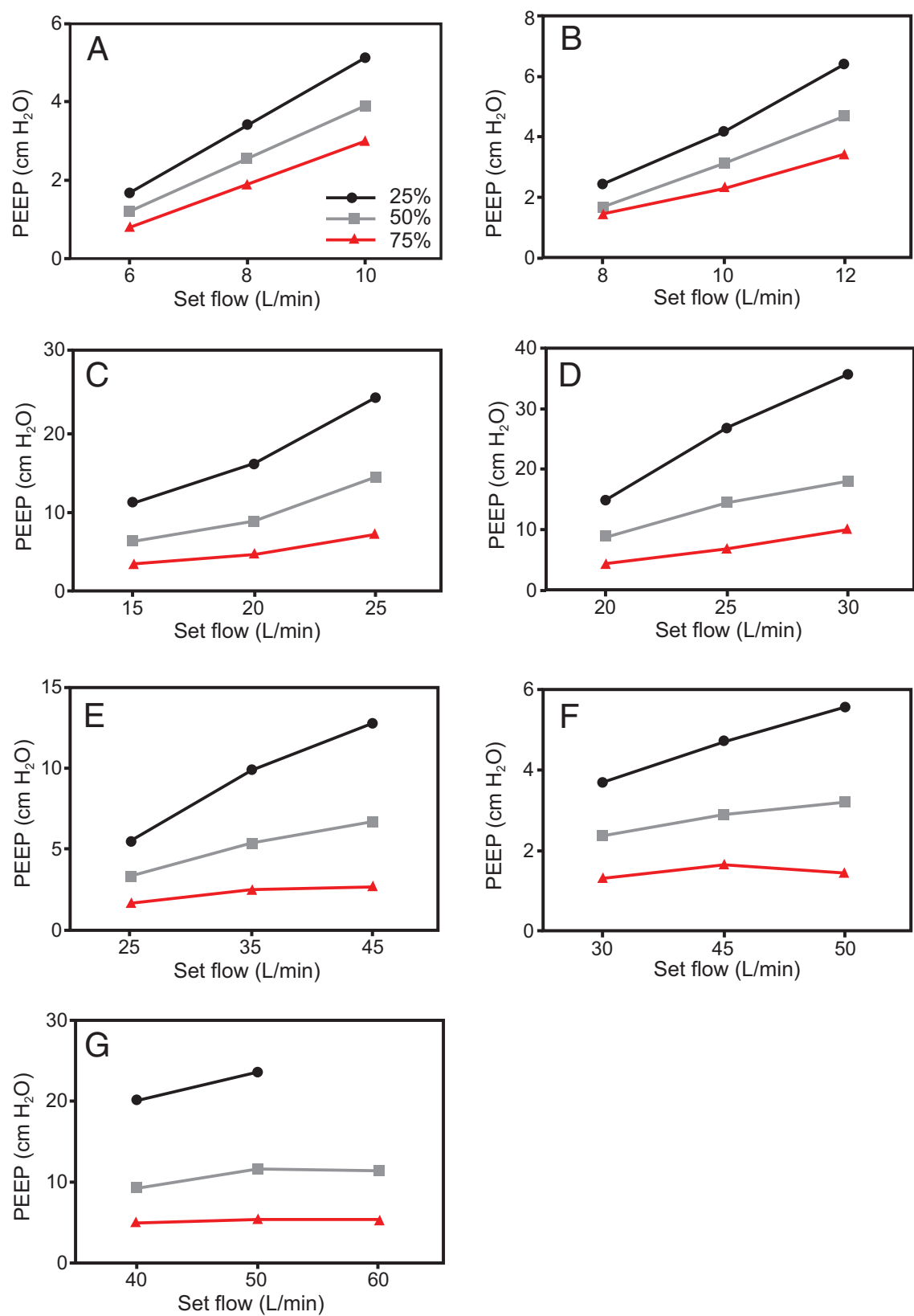

Fig. 3. Lung model alveolar end-expiratory pressure or PEEP as a function of high-flow nasal cannula set flow with various leaks $(25 \%$ leak, $50 \%$ leak, and $75 \%$ leak). Each curve represents a different leak. Individual data points are shown for each pressure measurement. Mouth leak is equal to indicated percentages of the set flow.

a $25 \%$ leak for the 1-month-old, 6-month-old, 1-y-old, and 3 -y-old models. This pressure increase was magnified as the HFNC flow increased (Fig. 3).

Intra-cannula pressure varied across trials as shown in Figure S1 (see the supplementary materials at http:// www.rcjournal.com). The intra-cannula pressure increased with an increase in flow and decreased with an increase in leak. The younger simulated models (ie, 1-month-old, 6-month-old, 1-y-old, and 3-y-old) produced very high intra-cannula pressures compared to the older simulated models (ie, 6-y-old, 12-y-old, and 18-y-old) $(P<.001$ and $>10 \%$ difference). This was likely due to the small crosssectional area of the HFNC circuit used and the nares of the individual models. Specifically, the younger simulated models used infant- and pediatric-sized cannulas, whereas the older simulated models used adult-sized cannulas (Table 2 ). The end-expiratory alveolar pressure changes with increasing flows and increasing leaks are shown in detail in Figure 3 and Table S1. Although not shown, the tracheal pressures trended similarly throughout all simulated mod- 
HFNC PEEP Generation in a Pediatric Model

Table 3. Mean Differences and 99.9\% CIs for Each Trial and Each Leak Setting

\begin{tabular}{|c|c|c|c|c|c|}
\hline \multirow{2}{*}{ Trial, Cannula Size (Flow) } & \multirow{2}{*}{ Leak, \% } & \multirow{2}{*}{ Leak, \% } & \multirow{2}{*}{ Mean Difference* } & \multicolumn{2}{|c|}{$99.9 \% \mathrm{CI}$} \\
\hline & & & & Lower Bound & Upper Bound \\
\hline \multirow[t]{6}{*}{1 month $(6 \mathrm{~L} / \mathrm{min})$} & 25 & 50 & 0.49 & 0.43 & 0.54 \\
\hline & & 75 & 0.89 & 0.84 & 0.95 \\
\hline & 50 & 25 & -0.49 & -0.54 & -0.43 \\
\hline & & 75 & 0.41 & 0.35 & 0.46 \\
\hline & 75 & 25 & -0.89 & -0.95 & -0.84 \\
\hline & & 50 & -0.41 & -0.46 & -0.35 \\
\hline \multirow[t]{6}{*}{1 month $(8 \mathrm{~L} / \mathrm{min})$} & 25 & 50 & 0.90 & 0.85 & 0.95 \\
\hline & & 75 & 1.56 & 1.51 & 1.61 \\
\hline & 50 & 25 & -0.90 & -0.95 & -0.85 \\
\hline & & 75 & 0.66 & 0.61 & 0.71 \\
\hline & 75 & 25 & -1.56 & -1.61 & -1.51 \\
\hline & & 50 & -0.66 & -0.71 & -0.61 \\
\hline \multirow[t]{6}{*}{1 month $(10 \mathrm{~L} / \mathrm{min})$} & 25 & 50 & 1.30 & 1.26 & 1.35 \\
\hline & & 75 & 2.20 & 2.16 & 2.25 \\
\hline & 50 & 25 & -1.30 & -1.35 & -1.26 \\
\hline & & 75 & 0.90 & 0.85 & 0.94 \\
\hline & 75 & 25 & -2.20 & -2.25 & -2.16 \\
\hline & & 50 & -0.90 & -0.94 & -0.85 \\
\hline \multirow[t]{6}{*}{6 month $(8 \mathrm{~L} / \mathrm{min})$} & 25 & 50 & 0.81 & 0.80 & 0.82 \\
\hline & & 75 & 1.22 & 1.21 & 1.23 \\
\hline & 50 & 25 & -0.81 & -0.82 & -0.80 \\
\hline & & 75 & 0.41 & 0.40 & 0.42 \\
\hline & 75 & 25 & -1.22 & -1.23 & -1.21 \\
\hline & & 50 & -0.41 & -0.42 & -0.40 \\
\hline \multirow[t]{6}{*}{6 month $(10 \mathrm{~L} / \mathrm{min})$} & 25 & 50 & 1.07 & 1.03 & 1.11 \\
\hline & & 75 & 1.92 & 1.88 & 1.96 \\
\hline & 50 & 25 & -1.07 & -1.11 & -1.03 \\
\hline & & 75 & 0.85 & 0.81 & 0.89 \\
\hline & 75 & 25 & -1.92 & -1.96 & -1.88 \\
\hline & & 50 & -0.85 & -0.89 & -.81 \\
\hline \multirow{6}{*}{6 month $(12 \mathrm{~L} / \mathrm{min})$} & 25 & 50 & 1.74 & 1.73 & 1.75 \\
\hline & & 75 & 3.02 & 3.01 & 3.03 \\
\hline & 50 & 25 & -1.74 & -1.75 & -1.73 \\
\hline & & 75 & 1.28 & 1.27 & 1.29 \\
\hline & 75 & 25 & -3.02 & -3.03 & -3.01 \\
\hline & & 50 & -1.28 & -1.29 & -1.27 \\
\hline \multirow[t]{6}{*}{1 y $(15 \mathrm{~L} / \mathrm{min})$} & 25 & 50 & 4.72 & 4.46 & 4.97 \\
\hline & & 75 & 7.55 & 7.30 & 7.80 \\
\hline & 50 & 25 & -4.72 & -4.97 & -4.46 \\
\hline & & 75 & 2.83 & 2.58 & 3.09 \\
\hline & 75 & 25 & -7.55 & -7.80 & -7.30 \\
\hline & & 50 & -2.83 & -3.09 & -2.58 \\
\hline \multirow[t]{6}{*}{1 y $(20 \mathrm{~L} / \mathrm{min})$} & 25 & 50 & 7.35 & 7.31 & 7.40 \\
\hline & & 75 & 11.39 & 11.35 & 11.44 \\
\hline & 50 & 25 & -7.35 & -7.40 & -7.31 \\
\hline & & 75 & 4.04 & 4.03 & 4.06 \\
\hline & 75 & 25 & -11.39 & -11.44 & -11.35 \\
\hline & & 50 & -4.04 & -4.06 & -4.03 \\
\hline \multirow[t]{6}{*}{1 y $(25 \mathrm{~L} / \mathrm{min})$} & 25 & 50 & 10.19 & 10.16 & 10.22 \\
\hline & & 75 & 17.27 & 17.24 & 17.30 \\
\hline & 50 & 25 & -10.19 & -10.22 & -10.16 \\
\hline & & 75 & 7.09 & 7.06 & 7.12 \\
\hline & 75 & 25 & -17.27 & -17.30 & -17.24 \\
\hline & & 50 & -7.09 & -7.12 & $\begin{array}{c}-7.06 \\
\text { (continued) }\end{array}$ \\
\hline
\end{tabular}


HFNC PEEP Generation in a Pediatric Model

Table 3. Continued

\begin{tabular}{|c|c|c|c|c|c|}
\hline \multirow{2}{*}{ Trial, Cannula Size (Flow) } & \multirow{2}{*}{ Leak, \% } & \multirow{2}{*}{ Leak, \% } & \multirow{2}{*}{ Mean Difference* } & \multicolumn{2}{|c|}{$99.9 \% \mathrm{CI}$} \\
\hline & & & & Lower Bound & Upper Bound \\
\hline \multirow[t]{6}{*}{3 y (20 L/min) } & 25 & 50 & 6.02 & 5.82 & 6.21 \\
\hline & & 75 & 10.60 & 10.40 & 10.80 \\
\hline & 50 & 25 & -6.02 & -6.21 & -5.82 \\
\hline & & 75 & 4.58 & 4.38 & 4.78 \\
\hline & 75 & 25 & -10.60 & -10.80 & -10.40 \\
\hline & & 50 & -4.58 & -4.78 & -4.38 \\
\hline \multirow[t]{6}{*}{3 y $(25 \mathrm{~L} / \mathrm{min})$} & 25 & 50 & 13.12 & 11.98 & 14.25 \\
\hline & & 75 & 20.51 & 19.38 & 21.65 \\
\hline & 50 & 25 & -13.12 & -14.25 & -11.98 \\
\hline & & 75 & 7.40 & 6.27 & 8.53 \\
\hline & 75 & 25 & -20.51 & -21.65 & -19.38 \\
\hline & & 50 & -7.40 & -8.53 & -6.27 \\
\hline \multirow[t]{6}{*}{3 y $(30 \mathrm{~L} / \mathrm{min})$} & 25 & 50 & 18.30 & 17.92 & 18.68 \\
\hline & & 75 & 26.48 & 26.10 & 26.87 \\
\hline & 50 & 25 & -18.30 & -18.68 & -17.92 \\
\hline & & 75 & 8.19 & 7.81 & 8.57 \\
\hline & 75 & 25 & -26.48 & -26.87 & -26.10 \\
\hline & & 50 & -8.19 & -8.57 & -7.81 \\
\hline \multirow[t]{6}{*}{6 y (25 L/min) } & 25 & 50 & 2.16 & 2.12 & 2.20 \\
\hline & & 75 & 3.94 & 3.91 & 3.98 \\
\hline & 50 & 25 & -2.16 & -2.20 & -2.12 \\
\hline & & 75 & 1.78 & 1.74 & 1.82 \\
\hline & 75 & 25 & -3.94 & -3.98 & -3.91 \\
\hline & & 50 & -1.78 & -1.82 & -1.74 \\
\hline \multirow[t]{6}{*}{6 y $(35 \mathrm{~L} / \mathrm{min})$} & 25 & 50 & 4.57 & 4.55 & 4.59 \\
\hline & & 75 & 7.54 & 7.52 & 7.57 \\
\hline & 50 & 25 & -4.57 & -4.59 & -4.55 \\
\hline & & 75 & 2.97 & 2.95 & 3.00 \\
\hline & 75 & 25 & -7.54 & -7.57 & -7.52 \\
\hline & & 50 & -2.97 & -3.00 & -2.95 \\
\hline \multirow[t]{6}{*}{6 y $(45 \mathrm{~L} / \mathrm{min})$} & 25 & 50 & 6.23 & 6.19 & 6.27 \\
\hline & & 75 & 10.41 & 10.38 & 10.45 \\
\hline & 50 & 25 & -6.23 & -6.27 & -6.19 \\
\hline & & 75 & 4.19 & 4.15 & 4.23 \\
\hline & 75 & 25 & -10.41 & -10.45 & -10.38 \\
\hline & & 50 & -4.19 & -4.23 & -4.15 \\
\hline \multirow[t]{6}{*}{12 y $(30 \mathrm{~L} / \mathrm{min})$} & 25 & 50 & 1.36 & 1.35 & 1.37 \\
\hline & & 75 & 2.51 & 2.49 & 2.52 \\
\hline & 50 & 25 & -1.36 & -1.37 & -1.35 \\
\hline & & 75 & 1.15 & 1.13 & 1.16 \\
\hline & 75 & 25 & -2.51 & -2.52 & -2.49 \\
\hline & & 50 & -1.15 & -1.16 & -1.13 \\
\hline \multirow[t]{6}{*}{12 y (40 L/min) } & 25 & 50 & 1.89 & 1.88 & 1.90 \\
\hline & & 75 & 3.198 & 3.18 & 3.20 \\
\hline & 50 & 25 & -1.89 & -1.90 & -1.88 \\
\hline & & 75 & 1.30 & 1.29 & 1.31 \\
\hline & 75 & 25 & -3.19 & -3.20 & -3.18 \\
\hline & & 50 & -1.30 & -1.31 & -1.29 \\
\hline \multirow{6}{*}{12 y $(50 \mathrm{~L} / \mathrm{min})$} & 25 & 50 & 2.41 & 2.39 & 2.42 \\
\hline & & 75 & 4.27 & 4.26 & 4.29 \\
\hline & 50 & 25 & -2.41 & -2.42 & -2.39 \\
\hline & & 75 & 1.87 & 1.85 & 1.88 \\
\hline & 75 & 25 & -4.27 & -4.29 & -4.26 \\
\hline & & 50 & -1.87 & -1.88 & $\begin{array}{c}-1.85 \\
\text { (continued) }\end{array}$ \\
\hline
\end{tabular}


Table 3. Continued

\begin{tabular}{|c|c|c|c|c|c|}
\hline \multirow{2}{*}{ Trial, Cannula Size (Flow) } & \multirow{2}{*}{ Leak, \% } & \multirow{2}{*}{ Leak, \% } & \multirow{2}{*}{ Mean Difference* } & \multicolumn{2}{|c|}{$99.9 \% \mathrm{CI}$} \\
\hline & & & & Lower Bound & Upper Bound \\
\hline \multirow[t]{6}{*}{18 y (40 L/min) } & 25 & 50 & 10.99 & 10.58 & 11.41 \\
\hline & & 75 & -3.61 & -4.02 & -3.20 \\
\hline & 50 & 25 & -10.99 & -11.41 & -10.58 \\
\hline & & 75 & -14.60 & -15.02 & -14.19 \\
\hline & 75 & 25 & 3.61 & 3.20 & 4.02 \\
\hline & & 50 & 14.60 & 14.19 & 15.02 \\
\hline \multirow[t]{6}{*}{18 y (50 L/min) } & 25 & 50 & 12.22 & 11.82 & 12.62 \\
\hline & & 75 & 18.44 & 18.04 & 18.84 \\
\hline & 50 & 25 & -12.22 & -12.62 & -11.82 \\
\hline & & 75 & 6.21 & 5.81 & 6.61 \\
\hline & 75 & 25 & -18.44 & -18.84 & -18.04 \\
\hline & & 50 & -6.21 & -6.61 & -5.81 \\
\hline \multirow[t]{2}{*}{18 y $(60 \mathrm{~L} / \mathrm{min})$} & 50 & 75 & 6.18 & 6.17 & 6.20 \\
\hline & 75 & 50 & 6.18 & 6.16 & 6.20 \\
\hline
\end{tabular}

els (Table S2; see the supplementary materials at http:// www.rcjournal.com). Because exhalation was passive, expiratory muscle pressure at end-exhalation was always zero (Table S2).

\section{Discussion}

There were four primary findings in our study. We reported that increasing the HFNC flows resulted in increasing end-expiratory alveolar pressure delivered at all measured flows. In addition, increasing the leak in the circuit resulted in decreased end-expiratory alveolar pressure at all measured flows. End-expiratory alveolar pressure delivered varied depending on the pediatric model size and HFNC size. Last, the Comfort Flo HFNC and our institutional HFNC delivery system functioned comparably to other commercially available systems.

Our data on the development of PEEP with the HFNC is consistent with that published by other groups. Volsko et $\mathrm{al}^{30}$ reported that nasal cannula flows of $2-6 \mathrm{~L} / \mathrm{min}$ did not generate $\mathrm{PEEP}>1.3 \mathrm{~cm} \mathrm{H}_{2} \mathrm{O}$ in an infant airway model in which they did not utilize leak percentage. In our model, the measured PEEP of $1.2 \mathrm{~cm} \mathrm{H}_{2} \mathrm{O}$ at 6 $\mathrm{L} / \mathrm{min}$ with $75 \%$ leak corresponded to these findings. Luo et al ${ }^{31}$ measured the PEEP generated with 3 different HFNC devices at flows of $0-80 \mathrm{~L} / \mathrm{min}$ in what appears to be an adult model with a closed-mouth and open-mouth leak and undescribed HFNC cannula sizes. They demonstrated that flows of $60 \mathrm{~L} / \mathrm{min}$ delivered 4-10 $\mathrm{cm} \mathrm{H}_{2} \mathrm{O}$ of PEEP in their airway model. The marked difference in the maximum PEEP measured compared to our model is likely due to differing leak percentages and different interfaces between HFNC device and the airway model nares.

\section{Generation of PEEP}

Comparatively, a study by Lavizzari et $\mathrm{al}^{32}$ reported that HFNC flows of 2-7 L/min generated PEEPs of 2-4 $\mathrm{cm} \mathrm{H}_{2} \mathrm{O}$ as measured with esophageal manometry in preterm infants when their mouths were closed. Furthermore, they demonstrated that the work of breathing required in HFNC and nasal PEEP were comparable. ${ }^{32}$ This study accounted for the interface of the patient nares to the HFNC device, standardizing the patients to an appropriate size.

In review of adult post-cardiac surgery patients, Parke et $\mathrm{al}^{7}$ reported that, at $35 \mathrm{~L} / \mathrm{min}$ of flow with a single size of HFNC, an average of $2.7 \mathrm{~cm} \mathrm{H}_{2} \mathrm{O}$ of PEEP was delivered. ${ }^{7}$ Although differing leak percentages and different size cannulas were not used in this study, similar PEEP was delivered at similar HFNC flows in the airway models used in our study (Fig. 3). Other studies in adult patients and airway models have reported that PEEP delivered by different HFNC devices at different flows is similar to the results from our airway models at the corresponding flows. ${ }^{1,2,5,7,9-11,14,16,31,32}$

\section{Effect of Leak Size}

Several studies in neonates have reported a decrease in PEEP generated with open-mouth breathing. Luo et $\mathrm{al}^{31}$ noted that the measured PEEP became negligible when the patient's mouth was open. Other studies have acknowledged the decrease in PEEP with open-mouth breathing or leaks in the flow delivery system, but to our knowledge no studies have measured specific changes in PEEP delivered with incremental, measured leak percentages.

Previous studies have reported that open-mouth breathing increases the air leak in the HFNC system and results 
in less PEEP delivered in adult subjects. ${ }^{1,3,5,7,9,14,24,25,31,32}$ These consistent results are likely due to the adult subjects' ability to follow instructions. To our knowledge, the study by Nielsen et $\mathrm{al}^{10}$ is the only one to date that used pediatric models to demonstrate a decrease in PEEP of $\sim 50 \%$ when simulating an open mouth in their airway models.

\section{Impact of Patient and Cannula Size on PEEP}

Given that the HFNC is not a closed system, we hypothesized that, as the size of the simulated patients and cannulas changed, there would be marked changes in the amount of PEEP established. This is likely due to a nonocclusive interface between the cannulas and the airway model nares. This likely resulted in leakage from the system that was not measured by our ventilation tester. Other studies have also acknowledged that HFNC size relative to patient nares can affect the flow and thus the PEEP delivered.7,10,11,15,24,25,30,31 Unfortunately, there was no reliable way to measure these subtle differences. We suspect that the issue exists in clinical practice, adding to the inability to reasonably estimate the amount of PEEP established in any given patient at the alveolar level.

\section{Novelty of Our Findings}

This study demonstrates that the use of the Comfort Flo HFNC establishes PEEP levels equivalent to those obtained with the Optiflow and Precision Flow systems, and that our unique institutional high-flow delivery system performs in a manner equivalent to that of commercially available high-flow systems. We also noted that, at $25 \%$ leak in some weight ranges, there is a potential for dangerously high PEEP levels to be established during HFNC therapy. Finally, we assessed PEEP generated by the full range of flows used in pediatric patients and young adults at leak levels of $25 \%, 50 \%$, and $75 \%$ of total flow delivered.

\section{Impact on Practice}

HFNC has enhanced the care of patients with respiratory insufficiency in the pediatric ICU setting. Several studies have associated the use of HFNC with a decrease in the frequency of intubation of pediatric subjects. ${ }^{13,33,34}$ Avoiding intubation likely confers a variety of benefits, including more ventilator-free days, shorter length of stay in the ICU, and possibly even fewer complications, such as laryngotracheal injury and development of subglottic stenosis. ${ }^{16,35-38}$ Several mechanisms have been proposed by which HFNC reduces the work of breathing, including washout of nasopharyngeal dead space, improvement of airway conductance and pulmonary compliance by warm air humidification, and generating
PEEP to the lungs. , $2,5-11,13,14,16,31,32$ The major clinical impact of our finding is that it is impossible to know the level of PEEP applied to a particular patient with a HFNC system. Because leak flow can change very rapidly, it becomes impossible to know with any assurance the PEEP applied. This is especially true with infants and children 1-6 years old, in whom dangerously high PEEP levels may be applied if leak flow is reduced to $25 \%$. We also noted that, at leak flow of $25 \%$, the 18 -y-old patient model exhibited excessive PEEP. However, it is highly unlikely that PEEP would increase to this level because of the large oral leaks observed in patients of this size and the fact that clinical studies have indicated in adults that PEEP established with HFNC is $\sim 1 \mathrm{~cm} \mathrm{H}_{2} \mathrm{O}$ for every $10 \mathrm{~L} / \mathrm{min}$ of flow. ${ }^{1,5-9}$ Clearly, although difficult to obtain, more clinical data on the PEEP level established during HFNC application is needed. Because it is difficult to use esophageal cannulas in small pediatric patients, the use of lung ultrasound, diaphragm electrical activity, and electrical impedance tomography may provide better insight into the level of PEEP established during HFNC therapy.

\section{Limitations}

The primary limitation of this study is that it was not performed in pediatric patients but rather in models that simulated pediatric airways. However, we believe that the essential mechanics of the pediatric respiratory system are well represented by this model. Although the numbers may vary depending upon the length and resistance of a pediatric patient's respiratory tract, it is clear that HFNC establishes a variable level of PEEP at moderate flows (eg, $8-50 \mathrm{~L} / \mathrm{min})$.

\section{Conclusion}

HFNCs deliver varying amounts of PEEP at the alveolar level with flows of 6-60 L/min. Increasing flow and decreasing leak resulted in the generation of greater PEEP. PEEP levels differed across cannulas and model weights at the same leak level, likely related to differences in the nasal interface between the HFNC device and the model nares.

\section{REFERENCES}

1. Nishimura M. High-flow nasal cannula oxygen therapy in adults: physiological benefits, indication, clinical benefits, and adverse effects. Respir Care 2016;61(4):529-541.

2. Sztrymf B, Messika J, Bertrand F, Hurel D, Leon R, Dreyfuss D, et al. Beneficial effects of humidified high flow nasal oxygen in critical care patients: a prospective pilot study. Intensive Care Med 2011;37(11):1780-1786.

3. Frat JP, Brugiere B, Ragot S, Chatellier D, Veinstein A, Goudet V, et al. Sequential application of oxygen therapy via high-flow nasal 


\section{HFNC PEEP Generation in a Pediatric Model}

cannula and noninvasive ventilation in acute respiratory failure: an observational pilot study. Respir Care 2015;60(2):170-178.

4. Boyle M, Chaudhary R, Kent S, O'Hare S, Broster S, Dassios T. High-flow nasal cannula on transport: moving with the times. Acta Paediatr 2014;103(5):e181.

5. Papazian L, Corley A, Hess D, Fraser JF, Frat JP, Guitton C, et al. Use of high-flow nasal cannula oxygenation in ICU adults: a narrative review. Intensive Care Med 2016;42(9):1336-1349.

6. Mikalsen IB, Davis P, Oymar K. High flow nasal cannula in children: a literature review. Scand J Trauma Resusc Emerg Med 2016; 24:93.

7. Parke RL, Eccleston ML, McGuinness SP. The effects of flow on airway pressure during nasal high-flow oxygen therapy. Respir Care 2011;56(8):1151-1155.

8. Iyer NP, Mhanna MJ. Association between high-flow nasal cannula and end-expiratory esophageal pressures in premature infants. Respir Care 2016;61(3):285-290.

9. Parke RL, McGuinness SP. Pressures delivered by nasal high flow oxygen during all phases of the respiratory cycle. Respir Care 2013; 58(10): 1621-1624.

10. Nielsen KR, Ellington LE, Gray AJ, Stanberry LI, Smith LS, DiBlasi RM. Effect of high-flow nasal cannula on expiratory pressure and ventilation in infant, pediatric, and adult models. Respir Care 2018; 63(2):147-157.

11. Sivieri EM, Gerdes JS, Abbasi S. Effect of HFNC flow rate, cannula size, and nares diameter on generated airway pressures: an in vitro study. Pediatr Pulmonol 2013;48(5):506-514.

12. Gerhardt T, Hehre D, Feller R, Reifenberg L, Bancalari E. Pulmonary mechanics in normal infants and young children during first 5 years of life. Pediatr Pulmonol 1987;3(5):309-316.

13. McKiernan C, Chua LC, Visintainer PF, Allen H. High flow nasal cannulae therapy in infants with bronchiolitis. J Pediatr 2010;156(4): 634-638.

14. Nishimura M. High-flow nasal cannula oxygen therapy in adults. J Intensive Care 2015;3(1):15.

15. Phelan PD, Williams HE. Ventilatory studies in healthy infants. Pediatr Res 1969;3(5):425-432.

16. Tu G, He H, Yin K, Ju M, Zheng Y, Zhu D, et al. High-flow nasal cannula versus noninvasive ventilation for treatment of acute hypoxemic respiratory failure in renal transplant recipients. Transplant Proc 2017;49(6):1325-1330.

17. Solomon R, Jurica K. Closing the research-practice gap: increasing evidence-based practice for nasogastric tube insertion using education and an electronic order set. J Emerg Nurs 2017;43(2):133-137.

18. Ganti L. Atlas of Emergency Medicine Procedures. Springer-Verlag New York. 2016

19. Thomsen TW, Shaffer RW, Setnik GS. Videos in clinical medicine. Nasogastric intubation. N Engl J Med 2006;354(17):e16.

20. Stocks J. Effect of nasogastric tubes on nasal resistance during infancy. Arch Dis Child 1980;55(1):17-21.

21. Breatnach E, Abbott GC, Fraser RG. Dimensions of the normal human trachea. AJR Am J Roentgenol1984;142(5):903-906.

22. Dullenkopf A, Kretschmar O, Knirsch W, Tomaske M, Hug M, Stutz $\mathrm{K}$, et al. Comparison of tracheal tube cuff diameters with internal transverse diameters of the trachea in children. Acta Anaesthesiol Scand 2006;50(2):201-205.

23. Griscom NT, Wohl ME. Dimensions of the growing trachea related to age and gender. AJR 1986;146(2):233-237.

24. Itagaki T, Bennett DJ, Chenelle CT, Fisher DF, Kacmarek RM. Performance of Leak compensation in all-age ICU ventilators during volume-targeted neonatal ventilation: a lung model study. Respir Care 2017;62(1):10-21.

25. Itagaki T, Chenelle CT, Bennett DJ, Fisher DF, Kacmarek RM. Effects of leak compensation on patient-ventilator synchrony during premature/neonatal invasive and noninvasive ventilation: a lung model study. Respir Care 2017;62(1):22-33.

26. Likus W, Bajor G, Gruszczynska K, Baron J, Markowski J. Nasal region dimensions in children: a CT study and clinical implications. Biomed Res Int 2014;2014:125810.

27. Rusconi F, Castagneto M, Gagliardi L, Leo G, Pellegatta A, Porta N, et al. Reference values for respiratory rate in the first 3 years of life. Pediatrics 1994;94(3):350-355.

28. Sforza C, Grandi G, De Menezes M, Tartaglia GM, Ferrario VF. Age- and sex-related changes in the normal human external nose. Forensic Sci Int 2011;204(1-3):205.e1-9.

29. Weiler T, Kamerkar A, Hotz J, Ross P, Newth C, Khemani R. The relationship between high flow nasal cannula flow rate and effort of breathing in children. J Pediatrics 2017;189:66-71.e3.

30. Volsko TA, Fedor K, Amadei J, Chatburn RL. High flow through a nasal cannula and CPAP effect in a simulated infant model. Respir Care 2011;56(12):1893-1900.

31. Luo JC, Lu MS, Zhao ZH, Jiang W, Xu B, Weng L, et al. Positive end-expiratory pressure effect of 3 high-flow nasal cannula devices. Respir Care 2017;62(7):888-895.

32. Lavizzari A, Veneroni C, Colnaghi M, Ciuffini F, Zannin E, Fumagalli M, et al. Respiratory mechanics during NCPAP and HHHFNC at equal distending pressures. Arch Dis Child Fetal Neonatal Ed 2014;99(4):F315-20.

33. Schibler A, Pham TM, Dunster KR, Foster K, Barlow A, Gibbons K, et al. Reduced intubation rates for infants after introduction of highflow nasal prong oxygen delivery. Intensive Care Med 2011;37(5): 847-852.

34. Wing R, James C, Maranda LS, Armsby CC. Use of high-flow nasal cannula support in the emergency department reduces the need for intubation in pediatric acute respiratory insufficiency. Pediatr Emerg Care 2012;28(11):1117-1123.

35. Bishop MJ. Mechanisms of laryngotracheal injury following prolonged tracheal intubation. Chest 1989;96(1):185-186.

36. Riese J, Fierce J, Riese A, Alverson BK. Effect of a hospital-wide high-flow nasal cannula protocol on clinical outcomes and resource utilization of bronchiolitis patients admitted to the PICU. Hosp Pediatr 2015;5(12):613-618.

37. Schweiger C, Manica D, Pereira DRR, Carvalho PRA, Piva JP, Kuhl $\mathrm{G}$, et al. Undersedation is a risk factor for the development of subglottic stenosis in intubated children. J Pediatr (Rio J) 2017;93(4): 351-355.

38. Schweiger C, Marostica PJ, Smith MM, Manica D, Carvalho PR, Kuhl G. Incidence of post-intubation subglottic stenosis in children: prospective study. J Laryngol Otol 2013;127(4):399-403. 